\title{
Retrospective Analyses of the Clinicopathological and Prognostic Features of Esophageal Squamous Cell Carcinoma in a Grade A Tertiary Hospital in Xinjiang, China
}

\section{Lifei Yang}

First Affiliated Hospital of Xinjiang Medical University

\section{Shutao Zheng}

First Affiliated Hospital of Xinjiang Medical University

\section{Qing Liu}

First Affiliated Hospital of Xinjiang Medical University

Tao Liu

First Affiliated Hospital of Xinjiang Medical University

\section{Qiqi Zhang}

First Affiliated Hospital of Xinjiang Medical University

\section{Xiujuan Han}

First Affiliated Hospital of Xinjiang Medical University

\section{Aerziguli Tuerxun}

First Affiliated Hospital of Xinjiang Medical University

Xiaomei Lu ( $\square$ luxiaomei88@163.com )

First Affiliated Hospital of Xinjiang Medical University

\section{Research Article}

Keywords: Esophageal squamous cell carcinoma, Xinjiang, clinicopathological, prognosis

Posted Date: February 24th, 2021

DOI: https://doi.org/10.21203/rs.3.rs-225842/v1

License: (c) (i) This work is licensed under a Creative Commons Attribution 4.0 International License. Read Full License 


\section{Abstract}

Background: The aim of this invesigation was to retrospectively analyze and summarize the clinicopathological and prognostic features of patients with esophageal squamous cell carcinoma (ESCC) at the Cancer Hospital Affiliated of the Xinjiang Medical University , a Grade A tertiary hospital in Xinjiang.

Methods: The clinicopathological data and follow-up data were collected from 419 ESCC patients. The overall survival (OS) and the related factors affecting survival were analyzed.

Results: Among the patients , 265 (63.2\%) did not receive any postoperative treatment. The 1-, 3-, and 5year overall survival (OS) rates were $84.8 \%, 47.5 \%$, and $37.3 \%$, respectively, and the median survival time (MST) was 29.0 months . Multivariate analysis showed that sources of patients, tumor stage, lymph node metastasis and stage of the disease were the independent predictors of OS. The finding in subgroup analysis indicated that there was a positive relationship between area and overall survival.

Conclusions: Postoperative treatments of these patients with ESCC were shown to be inadequent. Postadjuvant therapies of patients need to be strengthened. Targeted treatment regimes of different regions should be carried out by health department to alleviate the cancer burden.

\section{Background}

In developing countries, esophageal cancer (EC) is associated with high mortality [1]. Accounting for more than $50 \%$ of the global morbidity and mortality, incidence rate of ESCC in China are in the top 5 regions worldwide [2]. The two major subtypes of EC are ESCC and adenocarcinoma (EAC). They are epidemiologically and histopathologically distinctive. In the world, ESCC accounts for 70\% of cases of EC globally, and is particularly common in northern China with annual incidence rates being 100 per 100,000 population $[3,4]$. The incidence rate of ESCC in northern China is much higher than the average rate in China with 18.85 per 100,000 and also the average global rate of ESCC with 3.2 per 100,000 [1]. High incidence rates of ESCC have been reported in Xinjiang Province, especially in the Kazakh population [2]. It has been reported that tobacco, alcohol[5], and genetic background[6] increases the risk to develop EC. Despite this, pre-identifying individuals at poor prognosis for ESCC remains a major public health problem.

Although the epidemiological investigation of Chen et al.[7] on cancer incidence and mortality rates for ESCC utilized the cancer registries of Xinyuan county in Xinjiang, their study failed to analyze the prognostic risk factors of ESCC in Xinjiang. In addition, large-scale studies on the prognosis of ESCC patients in Xinjiang are insufficient. Furthermore, according to epidemiological data, the incidence of EC declined from 2001 to 2011. Whether the changes of prognosis of ESCC were similarly changed is unknown. 
Currently, the main treatments for ESCC include surgery, chemotherapy and radiotherapy $[8,9]$. The indications for radiotherapy in ESCC were less strict than surgical treatment; however, local recurrence is the main reason of failure for ESCC treated by chemoradiotherapy alone[10]. Although Zhang et al. explored the differences in the curative and side effects of chemoradiotherapy on EC among Xinjiang Han, Uygur and Kazakh patients. But their sample size was not large enough, totaling 170 [11]. A piece of study has reported that 5-year OS of ESCC patients undergoing radiotherapy was about $10 \%$ [8].

Chemotherapy in ESCC is mainly used for advanced disease and distant metastasis, or used with surgery for combined therapy as auxiliary or palliative treatment. Surgery is still the primary treatment modality in most patients with ESCC.

Therefore, understanding the prognostic factors of patients undergoing surgery is of great significance for prevention and treatment of EC.Till now, few studies focused on the pathogenesis and epidemiology of EC and OS of surgery among Xinjiang. So it is necessary to make a large-scale epidemiological study of ESCC in Xinjiang. This study aimed to explore the clinical features and prognostic factors of surgical treatment in patients with ESCC at the Cancer Hospital Affiliated of Xinjiang Medical University.

\section{Methods}

This study was approved by the medical ethics committee of Affiliated Tumor Hospital of Xinjiang Medical University. A waiver of informed consent was granted because of the retrospective nature of the research. From January 2010 to December 2019, 419 patients with ESCC, who were hospitalized in the Cancer Hospital Affiliated of Xinjiang Medical University were enrolled in this study. All subjects were treated with surgical dissection and had complete clinical and follow-up data. All cases were pathologically confirmed with ESCC. Routine hematological, liver and renal function tests, lung functions, electrocardiogram, gastroendoscopy, and chest and abdominal computed tomography (CT) were routinely carried out preoperatively to rule out surgical contraindications and distant metastasis. Internal medicine diseases were treated before operation actively. Data including age, gender, history of alcohol use, history of smoking, sources of patients, postoperative pathological information and follow-up data were collected. Tumor staging was based on the 8th edition of TNM staging published in 2017. All methods were carried out in accordance with relevant guidelines and regulations from the medical ethics committee of Affiliated Tumor Hospital of Xinjiang Medical University.

\section{Statistics}

The qualitative data were expressed as percentages. The differences between the groups were assessed using Chi-square test (X2) or Fisher's Least Significant Difference test. Survival rates were analyzed using the Kaplan-Meier method. Univariate analyses were performed to identify prognostic variables related to OS. Univariate variables with probability values $<0.05$ were selected for inclusion in the multivariate Cox proportional hazard The STATA statistical software package version15.0 (STATA Corp., College Station, 
Texas, USA) was used for all statistical analyses. A p value of 0.05 or less was defined as statistically significant.

\section{Results}

\section{Patient baseline characteristics}

The baseline characteristics of these 419 patients were presented in Table 1. 253 patients $(60.38 \%)$ were more than 60 years old; and $68.26 \%$ of the patients were men. 151 and 98 subjects had smoking history and alcohol history, respectively. The tumor was located in the upper chest in 40 cases, in the middle chest in 217 cases, and in the lower chest in 162 cases. The most proportion of patients grade and TNM stage of tumors were moderate and III, respectively. 
Table 1

Clinicopathologic features of 419 patients of ESCC

\begin{tabular}{|c|c|}
\hline Characteristics & No.[\%] \\
\hline \multicolumn{2}{|l|}{ Age } \\
\hline$\leq 60$ & $166 \rrbracket 39.62 \% \rrbracket$ \\
\hline$\$ 60$ & $253 \rrbracket 60.38 \% \rrbracket$ \\
\hline \multicolumn{2}{|l|}{ Gender } \\
\hline Male & $286 \rrbracket 68.26 \% \rrbracket$ \\
\hline Female & $133 \varangle 31.74 \% \rrbracket$ \\
\hline \multicolumn{2}{|c|}{ History of smoking } \\
\hline Yes & $151(36.04 \%)$ \\
\hline No & $268(63.96 \%)$ \\
\hline \multicolumn{2}{|c|}{ History of alcohol use } \\
\hline Yes & $98(23.39 \%)$ \\
\hline No & $321(76.61 \%)$ \\
\hline \multicolumn{2}{|l|}{ Family history } \\
\hline Yes & $60(14.32 \%)$ \\
\hline No & $359(85.68 \%)$ \\
\hline \multicolumn{2}{|l|}{ Tumor location } \\
\hline Upper & $40(9.55 \%)$ \\
\hline Middle & $217(51.79 \%)$ \\
\hline Lower & 162(38.66\%) \\
\hline \multicolumn{2}{|l|}{ Tumor length } \\
\hline$\otimes 3 \mathrm{~cm}$ & $103(24.58 \%)$ \\
\hline$\geq 3 \mathrm{~cm}$ & $316(75.42 \%)$ \\
\hline \multicolumn{2}{|l|}{ Nerve invasion } \\
\hline Yes & $91(21.72 \%)$ \\
\hline No & $328(78.28 \%)$ \\
\hline Vascular cance & \\
\hline
\end{tabular}




\begin{tabular}{|c|c|}
\hline Yes & $82(19.57 \%)$ \\
\hline No & $337(80.43 \%)$ \\
\hline \multicolumn{2}{|c|}{ Thoracoscopy } \\
\hline Yes & $314(74.94 \%)$ \\
\hline No & $105(25.06 \%)$ \\
\hline \multicolumn{2}{|c|}{ Adjuvant therapy } \\
\hline Yes & 154囚36.75\%区 \\
\hline No & $265 \rrbracket 63.25 \% \rrbracket$ \\
\hline \multicolumn{2}{|c|}{ Sources of patients } \\
\hline Urban & $247(58.95 \%)$ \\
\hline Rural & 172(41.05\%) \\
\hline \multicolumn{2}{|l|}{ Nationality } \\
\hline Kazakh & $84 \llbracket 20.05 \% \rrbracket$ \\
\hline Others & $335 \bigotimes 79.95 \% \rrbracket$ \\
\hline \multicolumn{2}{|c|}{ Histologic grade } \\
\hline Well & $21(5.01 \%)$ \\
\hline Moderate & $279(66.59 \%)$ \\
\hline Poor & $119(28.40 \%)$ \\
\hline \multicolumn{2}{|l|}{ pT category } \\
\hline pTis-pT1 & 63(15.04\%) \\
\hline pT2 & $60(14.32 \%)$ \\
\hline pT3 & $254(60.62 \%)$ \\
\hline pT4 & $42(10.02 \%)$ \\
\hline \multicolumn{2}{|l|}{$p N$ category } \\
\hline pNO & 186(44.39\%) \\
\hline pN1 & $114(27.21 \%)$ \\
\hline pN2 & 82(19.57\%) \\
\hline pN3 & $37(8.83 \%)$ \\
\hline pTNM stage & \\
\hline
\end{tabular}




\begin{tabular}{|ll|}
\hline II & $55(13.13 \%)$ \\
\hline III & $136(32.46 \%)$ \\
\hline IV & $224(53.46 \%)$ \\
\hline Gross classification & $4(0.95 \%)$ \\
\hline Ulcerative type & $148(35.32 \%)$ \\
\hline Protrude type & $64(15.27 \%)$ \\
\hline Modullary type & $186(44.40 \%)$ \\
\hline Fungating type & $21(5.01 \%)$ \\
\hline
\end{tabular}

\section{Treatment after operation}

Among the 419 patients, 265 patients (63.2\%) did not receive any postoperative treatment. In 154 patients who received adjuvant therapy; 60 subjects (14.3\%) received adjuvant chemotherapy, 43 subjects $(10.3 \%)$ underwent adjuvant radiotherapy, and 51 patients $(12.2 \%)$ were exposed to chemotherapy and radiotherapy (Fig.1a). Chemotherapy regimens included in all treatments were counted. The primary regimen of chemotherapy was paclitaxel-platinum combined chemotherapy. Fluorouracil (5-FU)-based regimens was the next most common. A minority of patients received oral chemotherapeutic agents (Fig.1b).

\section{Survival and prognostic factors for ESCC}

Patients were followed until March 2020 or until death, if earlier. The 1-, 3-, and 5-year overall survival (OS) rates were $84.8 \%, 47.5 \%$, and $37.3 \%$, respectively, and the median survival time (MST) was 29.0 months (Fig.2). Univariate analysis showed that tumor length, nerve invasion, vascular cancer embolus, sources of patients, nationality, tumor differentiation, tumor stage, lymph node metastasis, stage of disease and pathological type were the factors affecting the OS (Table 2). Multivariate analysis showed that sources of patients, tumor stage, lymph node metastasis and stage of the disease were the independent predictors of OS (Table 3). 
Table 2

Univariate analysis of the overall survival of the 419 patients with esophageal squamous cell carcinoma

\begin{tabular}{cllll}
\hline Variable & $\begin{array}{l}\text { No.patients } \\
\text { (\%) }\end{array}$ & $\begin{array}{l}\text { 1-year } \\
\text { OS(\%) }\end{array}$ & $\begin{array}{l}\text { 3-year } \\
\text { OS(\%) }\end{array}$ & $\begin{array}{l}\text { 5-year OS } \\
\text { (\%) }\end{array}$ \\
\hline Age & & & & \\
\hline$\leq 60$ & $166 \varangle 39.62 \% \rrbracket$ & $87.33 \%$ & $47.75 \%$ & $36.01 \%$ \\
\hline 660 & $253 \varangle 60.38 \% \square$ & $83.03 \%$ & $47.31 \%$ & $38.36 \%$ \\
\hline
\end{tabular}

Gender

0.140

Male

$286 \otimes 68.26 \% \rrbracket$

$84.45 \%$

$45.50 \%$

$34.48 \%$

Female

$133 \varangle 31.74 \% \square \quad 85.52 \%$

$51.84 \%$

$43.44 \%$

Smoking history

Yes

$151(36.04 \%)$

$85.97 \%$

$268(63.96 \%) \quad 84.11 \%$

$44.92 \%$

$36.51 \%$

No

$268(63.96 \%)$

$48.83 \%$

$38.03 \%$

Alcohol history

Yes

98(23.39\%)

$87.72 \%$

$321(76.61 \%)$

$83.87 \%$

$37.25 \%$

$31.85 \%$

No

Family history

$50.52 \%$

$39.04 \%$

Yes

No

Tumor location

Upper third
Middle third
Lower third

Tumor length

$₫ 3 \mathrm{~cm}$

Nerve invasion

Yes
No
Vascular cancer
embolus

\begin{tabular}{|llll|}
\hline $60(14.32 \%)$ & $88.21 \%$ & $58.06 \%$ & $58.06 \%$ \\
\hline $359(85.68 \%)$ & $84.21 \%$ & $45.89 \%$ & $34.47 \%$ \\
\hline
\end{tabular}

0.391

0.059

0.050

0.253

\begin{tabular}{|llll}
\hline $40(9.55 \%)$ & $82.50 \%$ & $32.60 \%$ & $28.98 \%$ \\
\hline $217(51.79 \%)$ & $85.50 \%$ & $49.35 \%$ & $38.71 \%$ \\
\hline $162(38.66 \%)$ & $84.41 \%$ & $48.61 \%$ & $37.22 \%$ \\
\hline
\end{tabular}

0.001

\begin{tabular}{|llllll}
\hline $103(24.58 \%)$ & $90.21 \%$ & $63.08 \%$ & $48.98 \%$ & \\
\hline $316(75.42 \%)$ & $82.94 \%$ & $42.37 \%$ & $33.20 \%$ & \\
\hline & & & & 0.001 \\
\hline $91(21.72 \%)$ & $76.73 \%$ & $26.74 \%$ & 21.39 & \\
\hline $328(78.28 \%)$ & $87.04 \%$ & $53.36 \%$ & $40.42 \%$ & \\
\hline & & & & 0.001 \\
\hline
\end{tabular}




\begin{tabular}{|c|cccc|} 
Yes & $82(19.57 \%)$ & $75.45 \%$ & $28.66 \%$ & $19.67 \%$ \\
\hline No & $337(80.43 \%)$ & $87.08 \%$ & $52.06 \%$ & $41.54 \%$ \\
\hline
\end{tabular}

Thoracoscopy

\begin{tabular}{lllll} 
Yes & $314(74.94 \%)$ & $84.81 \%$ & $45.83 \%$ & $37.44 \%$ \\
\hline No & $105(25.06 \%)$ & $84.76 \%$ & $51.11 \%$ & $37.28 \%$ \\
\hline
\end{tabular}

Adjuvant therapy

Yes

Sources of patients

Sources of patients

Urban

Rural

\begin{tabular}{llll}
$247(58.95 \%)$ & $91.00 \%$ & $55.30 \%$ & $45.60 \%$ \\
\hline $172(41.05 \%)$ & $75.90 \%$ & $36.10 \%$ & $23.20 \%$
\end{tabular}

\begin{tabular}{|c|c|}
\hline $154 \llbracket 36.75 \% \rrbracket$ & $85.40 \%$ \\
\hline $265 \llbracket 63.25 \% \rrbracket$ & $84.41 \%$ \\
\hline
\end{tabular}

50

Nationality

Kazakh

Others

\section{Histologic grade}

Carcinoma in situ

$84 \llbracket 20.05 \% \rrbracket$

$335 \otimes 79.95 \% \bigotimes \quad 88.60 \%$

\begin{tabular}{l}
$80.7 \%$ \\
$88.60 \%$ \\
\hline
\end{tabular}

$43.33 \%$

$50.05 \%$

$34.40 \%$

$39.30 \%$

0.943

\begin{tabular}{|c|c|c|c|c|c|}
\hline Well & $14(3.43 \%)$ & $85.71 \%$ & $33.33 \%$ & $33.33 \%$ & \\
\hline Moderate & $279(66.59 \%)$ & $87.28 \%$ & $48.97 \%$ & $40.17 \%$ & \\
\hline Poor & $119(28.40 \%)$ & $77.75 \%$ & $41.23 \%$ & $27.40 \%$ & \\
\hline pT category & & & & & 0.001 \\
\hline Tis & $4(0.95 \%)$ & $100 \%$ & $100 \%$ & - & \\
\hline T1 & $59 \rrbracket 14.09 \% \rrbracket$ & $95.00 \%$ & $85.21 \%$ & $66.46 \%$ & \\
\hline T2 & $60(14.32 \%)$ & $93.33 \%$ & $67.87 \%$ & $60.86 \%$ & \\
\hline T3 & $254(60.62 \%)$ & $82.41 \%$ & $36.84 \%$ & $27.80 \%$ & \\
\hline $\mathrm{T} 4$ & $42(10.02 \%)$ & $71.02 \%$ & $26.94 \%$ & $21.21 \%$ & \\
\hline$p N$ category & & & & & 0.001 \\
\hline pNO & $186(44.39 \%)$ & $93.00 \%$ & $70.77 \%$ & $55.94 \%$ & \\
\hline $\mathrm{pN} 1$ & $114(27.21 \%)$ & $81.91 \%$ & $39.21 \%$ & $30.97 \%$ & \\
\hline $\mathrm{pN} 2$ & $82(19.57 \%)$ & $76.40 \%$ & $21.77 \%$ & $16.63 \%$ & \\
\hline
\end{tabular}




\begin{tabular}{|clllll|}
\hline pN3 & $37(8.83 \%)$ & $70.27 \%$ & $4.94 \%$ & $3.63 \%$ & \\
\hline pTNM stage & & & & & 0.001 \\
\hline 0 & $4(0.96 \%)$ & $100 \%$ & $100 \%$ & - & \\
\hline I & $51(22.64 \%)$ & $94.21 \%$ & $89.64 \%$ & $65.61 \%$ & \\
\hline II & $136(32.54 \%)$ & $94.84 \%$ & $67.41 \%$ & $56.17 \%$ & \\
\hline III & $223(53.35 \%)$ & $76.42 \%$ & $24.67 \%$ & $19.03 \%$ & \\
\hline IV & $4(0.96 \%)$ & $75.00 \%$ & $75.00 \%$ & $75 \%$ & 0.004 \\
\hline Gross classification & & & & & \\
\hline Ulcerative type & $148(35.32 \%)$ & $84.85 \%$ & $43.23 \%$ & $31.62 \%$ & \\
\hline Protrude type & $64(15.27 \%)$ & $90.63 \%$ & $69.94 \%$ & $51.41 \%$ & \\
\hline Modullary type & $186(44.40 \%)$ & $82.57 \%$ & $42.46 \%$ & $35.98 \%$ & \\
\hline Fungating type & $21(5.01 \%)$ & $85.71 \%$ & $51.95 \%$ & $40.40 \%$ & \\
\hline
\end{tabular}

Table 3

Multivariate Cox proportional hazards regression models were used to evaluate the overall survival in subgroup analyses based on different clinicopathological features.

\begin{tabular}{|c|c|c|}
\hline \multirow[t]{2}{*}{ Variable } & \multicolumn{2}{|l|}{ Multivariable } \\
\hline & Odds ratio(95\%Cl) & $P$ value \\
\hline Tumor length & $0.763(0.525-1.110)$ & 0.158 \\
\hline Nerve invasion & $1.228 \otimes 0.903-1.670 \rrbracket$ & 0.190 \\
\hline Vascular cancer embolus & $1.078 \otimes 0.785-1.479 \rrbracket$ & 0.641 \\
\hline Source of patients & 1.747ه1.349-2.263凶 & 0.001 \\
\hline Nationality & $1.176 \bowtie 0.863-1.602 \rrbracket$ & 0.303 \\
\hline Histologic grade & $1.093(0.845-1.415)$ & 0.495 \\
\hline Gross classification & 1.039ه0.911-1.185》 & 0.563 \\
\hline T stage & $1.389(1.118-1.276)$ & 0.003 \\
\hline $\mathrm{N}$ stage & $1.363(1.154-1.612)$ & 0.001 \\
\hline TNM stage & $1.585(1.151-2.183)$ & 0.005 \\
\hline Histologic grade & $1.093(0.845-1.415)$ & 0.495 \\
\hline
\end{tabular}




\section{Subgroup analyses of sources of patients on different clinicopathological features and OS}

Considering sources of patients was the dominating independent prognostic factors, a further analysis assessed the clinicopathological significance of sources of patients was performed. Compared with the urban subjects, the rural patients were older $\left(X^{2}=4.006 \rrbracket p=0.045\right.$, Table 4$)$. The rural patients using thoracoscope were present in low proportions $\left(X^{2}=14.976 \rrbracket p \rrbracket 0.001\right.$, Table 4). Furthermore, the proportion of Kazakh patients in rural areas was significantly higher than that in urban areas $\left(X^{2}=8.163 \rrbracket p=0.004\right.$, Table 4). Finally, we assessed the influence of sources of patients on OS. No significant difference in OS was found in most subgroups, except for the nationality in patients from rural area (Table 5). In patients from urban area, history of drinking, tumor length, nerve invasion and vascular tumor thrombus were all the independent predictors of OS (Table 6). 
Table 4

Comparison of the clinicopathologic features between patients from urban area and patients from rural area

\begin{tabular}{|c|c|c|c|c|c|}
\hline & & Source of & patients & & \\
\hline Variable & No.patients (\%) & Urban & Rural & $x^{2}$ & $P$ value \\
\hline \multicolumn{6}{|l|}{ Age } \\
\hline$\leq 60$ & $166 \rrbracket 39.62 \% \rrbracket$ & 88 & 78 & 4.006 & 0.045 \\
\hline$\$ 60$ & $253 \rrbracket 60.38 \% \rrbracket$ & 159 & 94 & & \\
\hline \multicolumn{6}{|l|}{ Gender } \\
\hline Male & $286 \rrbracket 68.26 \% \rrbracket$ & 168 & 118 & 0.016 & 0.899 \\
\hline Female & $133 \rrbracket 31.74 \% \rrbracket$ & 79 & 54 & & \\
\hline \multicolumn{6}{|c|}{ Smoking history } \\
\hline Yes & $151(36.04 \%)$ & 95 & 56 & 1.533 & 0.216 \\
\hline No & $268(63.96 \%)$ & 152 & 116 & & \\
\hline \multicolumn{6}{|c|}{ Alcohol history } \\
\hline Yes & $98 \rrbracket 23.39 \% \rrbracket$ & 62 & 36 & 0.984 & 0.321 \\
\hline No & $321(76.61 \%)$ & 185 & 136 & & \\
\hline \multicolumn{6}{|c|}{ Family history } \\
\hline Yes & $60(14.32 \%)$ & 37 & 23 & 0.214 & 0.644 \\
\hline No & $359(85.68 \%)$ & 210 & 149 & & \\
\hline \multicolumn{6}{|c|}{ Tumor lacation } \\
\hline Upper & $40(9.55 \%)$ & 21 & 19 & 1.062 & 0.588 \\
\hline Middle & $217(51.79 \%)$ & 132 & 85 & & \\
\hline Lower & $162(38.66 \%)$ & 94 & 68 & & \\
\hline \multicolumn{6}{|c|}{ Tumor length } \\
\hline$\varangle 3 \mathrm{~cm}$ & $103(24.58 \%)$ & 73 & 30 & 1.183 & 0.277 \\
\hline$\geq 3 \mathrm{~cm}$ & $316(75.42 \%)$ & 174 & 141 & & \\
\hline \multicolumn{6}{|c|}{ Nerve invasion } \\
\hline Yes & $91(21.72 \%)$ & 52 & 39 & 0.157 & 0.692 \\
\hline No & $328(78.28 \%)$ & 195 & 133 & & \\
\hline
\end{tabular}


Vascular cancer embolus

\begin{tabular}{cccccc} 
Yes & $82(19.57 \%)$ & 46 & 36 & 0.343 & 0.588 \\
No & $337(80.43 \%)$ & 201 & 136 & & \\
\hline
\end{tabular}

Thoracoscopy

Yes

$314(74.94 \%) \quad 202$

112

$14.976 \quad \otimes 0.001$

No

105(25.06\%)

44

59

Adjuvant therapy

Yes

$154 \llbracket 36.75 \% \rrbracket$

82

72

$3.273 \quad 0.07$

No

$265 \rrbracket 63.25 \% \rrbracket$

165

100

Nationality

Kazakh

$84 \rrbracket 20.05 \% \bigotimes$

38

46

8.163

0.004

Others

$335 \rrbracket 79.95 \% \rrbracket$

209

126

Histologic grade

\begin{tabular}{|clllll|}
\hline Carcinoma in situ & $7(1.67 \%)$ & 4 & 3 & 0.224 & 0.974 \\
\hline Well & $14(3.43 \%)$ & 9 & 5 & & \\
\hline Moderate & $279(66.59 \%)$ & 165 & 114 & & \\
\hline Poor & $119(28.40 \%)$ & 69 & 50 & & \\
\hline pT category & & & & & \\
\hline Tis-T1 & $63(15.04 \% \bigotimes$ & 42 & 21 & 3.237 & 0.357 \\
\hline T2 & $60(14.32 \%)$ & 37 & 23 & & \\
\hline T3 & $254(60.62 \%)$ & 147 & 107 & & \\
\hline T4 & $42(10.02 \%)$ & 21 & 21 & & \\
\hline pN category & & & & & \\
\hline pN0 & $186(44.39 \%)$ & 119 & 67 & 4.344 & 0.227 \\
\hline pN1 & $114(27.21 \%)$ & 62 & 50 & & \\
\hline pN2 & $82(19.57 \%)$ & 42 & 40 & & \\
\hline pN3 & $37(8.83 \%)$ & 22 & 15 & & \\
\hline pTNM stage & & & & & \\
\hline 0 & $4(0.96 \%)$ & 3 & 1 & 0.224 & \\
\hline & & & & & \\
\hline
\end{tabular}




\begin{tabular}{|llllll|}
\hline I II & $51(12.17 \%)$ & 38 & 13 & & \\
III & $136(32.45 \%)$ & 81 & 55 & & \\
\hline IV & $225(53.70 \%)$ & 123 & 102 & & \\
\hline Gross classification & $3(0.72 \%)$ & 2 & 1 & & \\
\hline Ulcerative type & $148(35.32 \%)$ & 88 & 60 & 5.253 & 0.154 \\
\hline Protrude type & $64(15.27 \%)$ & 45 & 19 & & \\
\hline Modullary type & $186(44.39 \%)$ & 104 & 82 & & \\
\hline Fungating type & $21(5.01 \%)$ & 10 & 11 & & \\
\hline
\end{tabular}


Table 5

Univariate analysis of the overall survival of the 172 patients with esophageal squamous cell carcinoma from rural area

\begin{tabular}{|c|c|c|c|c|c|}
\hline Variable & $\begin{array}{l}\text { No.patients } \\
(\%)\end{array}$ & $\begin{array}{l}\text { 1-year } \\
\text { OS(\%) }\end{array}$ & $\begin{array}{l}\text { 3-year } \\
\text { OS(\%) }\end{array}$ & $\begin{array}{l}\text { 5-year OS } \\
\text { (\%) }\end{array}$ & $\begin{array}{l}P \\
\text { value }\end{array}$ \\
\hline Age & & & & & 0.592 \\
\hline$\leq 60$ & $78 \llbracket 45.35 \% \rrbracket$ & $84.60 \%$ & $39.60 \%$ & $21.90 \%$ & \\
\hline$\$ 60$ & $94 \rrbracket 54.65 \% \rrbracket$ & $77.30 \%$ & $33.00 \%$ & $26.30 \%$ & \\
\hline Gender & & & & & 0.931 \\
\hline Male & $78(45.35 \%)$ & $79.40 \%$ & $39.60 \%$ & $21.90 \%$ & \\
\hline Female & $94(54.65 \%)$ & $77.30 \%$ & $33.30 \%$ & $26.30 \%$ & \\
\hline Smoking history & & & & & 0.882 \\
\hline Yes & $56(32.56 \%)$ & $76.50 \%$ & $35.30 \%$ & $14.40 \%$ & \\
\hline No & $116(67.44 \%)$ & $75.50 \%$ & $35.20 \%$ & $21.30 \%$ & \\
\hline Alcohol history & & & & & 0.923 \\
\hline Yes & $36(20.93 \%)$ & $75.00 \%$ & $31.50 \%$ & $31.50 \%$ & \\
\hline No & 136(79.07\%) & $76.10 \%$ & $36.30 \%$ & $21.50 \%$ & \\
\hline Family history & & & & & 0.390 \\
\hline Yes & $23(13.37 \%)$ & $78.00 \%$ & $43.20 \%$ & $43.20 \%$ & \\
\hline No & 149(86.63\%) & $79.00 \%$ & $35.20 \%$ & $20.80 \%$ & \\
\hline Tumor lacation & & & & & 0.801 \\
\hline Upper third & $19(11.05 \%)$ & $78.90 \%$ & $21.40 \%$ & $21.40 \%$ & \\
\hline Middle third & $85(49.42 \%)$ & $72.40 \%$ & $35.30 \%$ & $16.00 \%$ & \\
\hline Lower third & $68(39.53 \%)$ & $79.30 \%$ & $25.60 \%$ & $25.60 \%$ & \\
\hline Tumor length & & & & & 0.079 \\
\hline$\otimes 3 \mathrm{~cm}$ & $31(18.02 \%)$ & $80.00 \%$ & $52.40 \%$ & $26.20 \%$ & \\
\hline$\geq 3 \mathrm{~cm}$ & $141(81.98 \%)$ & $77.00 \%$ & $32.60 \%$ & $21.60 \%$ & \\
\hline Nerve invasion & & & & & 0.191 \\
\hline Yes & $39(22.67 \%)$ & $71.40 \%$ & $23.10 \%$ & $23.10 \%$ & \\
\hline No & 133(77.33\%) & $77.20 \%$ & $39.80 \%$ & $23.10 \%$ & \\
\hline Vascular cancer embolus & & & & & 0.137 \\
\hline
\end{tabular}




\begin{tabular}{|c|c|c|c|c|c|}
\hline Yes & $36(20.93 \%)$ & $66.70 \%$ & $25.50 \%$ & $21.20 \%$ & \\
\hline No & $136(79.07 \%)$ & $78.40 \%$ & $38.80 \%$ & $24.10 \%$ & \\
\hline Thoracoscopy & & & & & 0.503 \\
\hline Yes & $112(65.12 \%)$ & $76.40 \%$ & $35.70 \%$ & $29.60 \%$ & \\
\hline No & $60(34.88 \%)$ & $76.30 \%$ & $34.80 \%$ & $20.40 \%$ & \\
\hline Adjuvant therapy & & & & & 0.450 \\
\hline Yes & $72 \rrbracket 41.86 \% \rrbracket$ & $84.40 \%$ & $38.50 \%$ & $28.30 \%$ & \\
\hline No & $100 \rrbracket 58.14 \% \rrbracket$ & $72.80 \%$ & $32.60 \%$ & $19.20 \%$ & \\
\hline Nationality & & & & & 0.01 \\
\hline Kazakh & $46 \rrbracket 20.05 \% \rrbracket$ & $64.30 \%$ & $19.40 \%$ & $11.70 \%$ & \\
\hline Others & 126ه79.95\%区 & $80.00 \%$ & $41.90 \%$ & $27.50 \%$ & \\
\hline
\end{tabular}


Table 6

Univariate analysis of the overall survival of the 247 patients with esophageal squamous cell carcinoma from urban area

\begin{tabular}{|c|c|c|c|c|c|}
\hline Variable & $\begin{array}{l}\text { No.patients } \\
(\%)\end{array}$ & $\begin{array}{l}\text { 1-year } \\
\text { OS(\%) }\end{array}$ & $\begin{array}{l}\text { 3-year } \\
\text { OS(\%) }\end{array}$ & $\begin{array}{l}\text { 5-year OS } \\
\text { (\%) }\end{array}$ & $\begin{array}{l}P \\
\text { value }\end{array}$ \\
\hline Age & & & & & 0.986 \\
\hline$\leq 60$ & $88 \llbracket 35.63 \% \rrbracket$ & $94.30 \%$ & $54.70 \%$ & $52.50 \%$ & \\
\hline$\triangle 60$ & $159 \bowtie 64.37 \% \rrbracket$ & $88.80 \%$ & $55.20 \%$ & $44.30 \%$ & \\
\hline Gender & & & & & 0.056 \\
\hline Male & $168(68.02 \%)$ & $89.2 \%$ & $52.10 \%$ & $54.00 \%$ & \\
\hline Female & $79(31.98 \%)$ & $94.80 \%$ & $62.30 \%$ & $26.30 \%$ & \\
\hline Smoking history & & & & & 0.148 \\
\hline Yes & $95(38.46 \%)$ & $91.80 \%$ & $50.60 \%$ & $30.70 \%$ & \\
\hline No & $152(61.54 \%)$ & $90.70 \%$ & $81.00 \%$ & $48.80 \%$ & \\
\hline Alcohol history & & & & & 0.005 \\
\hline Yes & $62(25.10 \%)$ & $75.00 \%$ & $40.70 \%$ & $23.90 \%$ & \\
\hline No & $185(74.90 \%)$ & $89.60 \%$ & $60.00 \%$ & $50.01 \%$ & \\
\hline Family history & & & & & 0.088 \\
\hline Yes & $37(14.98 \%)$ & $91.70 \%$ & $67.30 \%$ & $53.90 \%$ & \\
\hline No & $210(85.02 \%)$ & $90.40 \%$ & $53.40 \%$ & $42.40 \%$ & \\
\hline Tumor lacation & & & & & 0.278 \\
\hline Upper third & $21(8.50 \%)$ & $85.70 \%$ & $36.40 \%$ & $36.40 \%$ & \\
\hline Middle third & $132(53.44 \%)$ & $93.90 \%$ & $58.30 \%$ & $47.40 \%$ & \\
\hline Lower third & $94(38.06 \%)$ & $88.10 \%$ & $55.70 \%$ & $45.90 \%$ & \\
\hline Tumor length & & & & & 0.008 \\
\hline$\nabla 3 \mathrm{~cm}$ & $73(29.55 \%)$ & $94.40 \%$ & $67.50 \%$ & $56.80 \%$ & \\
\hline$\geq 3 \mathrm{~cm}$ & 174(70.45\%) & $89.50 \%$ & $50.10 \%$ & $40.60 \%$ & \\
\hline Nerve invasion & & & & & $\varangle 0.001$ \\
\hline Yes & $52(21.05 \%)$ & $80.70 \%$ & $29.10 \%$ & $19.40 \%$ & \\
\hline No & 195(78.95\%) & $93.80 \%$ & $62.60 \%$ & $50.40 \%$ & \\
\hline Vascular cancer embolus & & & & & $\nabla 0.001$ \\
\hline
\end{tabular}




\begin{tabular}{|c|c|c|c|c|c|}
\hline Yes & $46(18.62 \%)$ & $82.40 \%$ & $31.60 \%$ & $20.70 \%$ & \\
\hline No & 201(81.38\%) & $92.90 \%$ & $60.90 \%$ & $51.70 \%$ & \\
\hline Thoracoscopy & & & & & 0.065 \\
\hline Yes & 203(82.19\%) & $89.40 \%$ & $51.40 \%$ & $42.40 \%$ & \\
\hline No & $44(17.81 \%)$ & $97.70 \%$ & $70.50 \%$ & $56.50 \%$ & \\
\hline Adjuvant therapy & & & & & 0.154 \\
\hline Yes & $82 \rrbracket 33.20 \% \rrbracket$ & $90.00 \%$ & $47.50 \%$ & $35.40 \%$ & \\
\hline No & $165 \rrbracket 66.80 \% \rrbracket$ & $91.40 \%$ & $59.50 \%$ & $49.10 \%$ & \\
\hline Nationality & & & & & 0.344 \\
\hline Kazakh & $38 \rrbracket 15.38 \% \rrbracket$ & $92.00 \%$ & $46.50 \%$ & $31.0 \%$ & \\
\hline Others & $209 \rrbracket 84.62 \% \rrbracket$ & $90.80 \%$ & $57.00 \%$ & $46.40 \%$ & \\
\hline
\end{tabular}

\section{Discussion}

In this study, we retrospectively analyzed the data of 419 patients with ESCC at a single cancer center in Xinjiang area, which is one of the high-risk regions for esophageal cancer in China. The results showed that the adjuvant therapy for majority of patients were insufficiency by conducting postoperative therapy analyses. In addition to tumor stage, the regions of patients and nationality were also an independent prognostic factor. In further subgroup analysis, we confirmed that nationality was the main factor affecting the prognosis of patients from rural areas.

Our finding that ESCC patients had poor survival and our statistics indicating a lack of postoperative treatment revealed that multidisciplinary treatments, especially adjuvant therapy were essential for patients. Our results also revealed that the future management of these patients from rural area needed to be enhanced with further long-term follow-up data to support clinical decisions and interventional strategies. All of these will have a significant impact on the prognosis of patients by influencing the doctor's follow-up strategy.

In our study, most of them were male and over 60 years old. This is consistent with the results of epidemiological investigations that carcinomas of the esophagus in China occur mainly in people aged 60-74 years and the incidence of EC in men was twice as many as that in women' [7]. In our study, only about $23.6 \%$ of the patients were diagnosed with early stage 0 or $1 \mathrm{ESCC}$, and most of the patients were in middle or late stage. Our stage results are also consistent with the findings from other studies $[12,13]$. It has been acknowledged that morbidity of ESCC in the Kazakh population in Xinjiang was far higher 
than other ethnic minorities [14]. In our study, a worser prognosis was observed in Kazakh patients with ESCC. This is consistent with other study[14].

Additionally, in our multivariate analysis, we found that the source of patients is an important factor affecting the prognosis. This is in agreement with the previously reported data $[7,15,16]$. Kou et al. analyzed the possible reason for this discrepancy in their finding may lie in the difference of socioeconomic status (SES) disparities in the populations. Our results of subgroup analysis suggested that Kazakh patients from rural area even had a shorter prognosis than other populations living in the same area. This suggest that more attention should be paid to the sources of patients during clinical treatment.

In addition, our results suggested that the adjuvant therapy for majority of patients were insufficiency. The treatment of ESCC depends on the characteristics of the patient including health status and TNM stage. Current guidelines suggest additional treatment of patients with $\geq \mathrm{T} 2$ tumors, although the risk of node-negative T2 lesions is low [17]. The characteristics of esophageal resection alone can be considered ( $<2 \mathrm{~cm}$ and well-differentiated), but most subjects with esophageal cancer was locally advanced (>T2 and / or $\mathrm{N}+$ ) when they were diagnosed [17]. For these patients, to reduce primary tumor bulk chemotherapy, radiotherapy or chemo-radiotherapy isessential. Since about $87 \%$ of our patients were in middle or late stage, adjuvant therapy was recommended for most of them according to EC guidelines [18]; however, only 165 subjects received post-surgery treatments. Noticeably, we found that several patients did not receive complete postoperative treatment either for that they lived far from the hospital or for the side effects of the treatment. Our follow-up results revealed that postoperative management of patients with esophageal squamous cell carcinoma needs to be strengthened.

Our results showed that the 1-, 3-, and 5-year OS rates of this patients with ESCC were $84.8 \%, 47.5 \%$, and $37.3 \%$, which was worse than that was reported in a previous study [19]. In our univariate analysis of the overall survival of the 419 patients, tumor length, nerve invasion, vascular cancer embolus, sources of patients, nationality, tumor differentiation, tumor stage, lymph node metastasis, stage of disease and pathological type were related to OS. The association with tumor length, pT category, pN category, and TNM stage were consistent with the results of other studies [20-22]. It is accepted that smoking and drinking alcohol can increase the risk of ESCC from the results of a meta-analysis [23]. However, in our study, age, history of smoking, using thoracoscopy and region of patients were unrelated to their OS. Insufficient adjuvant therapy, diet or environmental factors may lead to lower OS. Therefore, they interferes with other prognostic factors. Some studies showed that older age was a prognostic factor [12, 13]. In our univariate analysis, age was not related to OS. Owing to improvements in surgical safety, age is less of a risk factor for the prognosis of ESCC. What's more, one of our findings disaccorded with others was that the OS of patients with a family history of cancer was longer than the OS of those with no history. This may be due to more active screening and treatments are conducted by patients with a family history of cancer. 
This study has some limitations. It is a single institution, retrospective study. The retrospective nature of this study may undermine its power. However, our cancer center is the largest in Xinjiang, which is one of the high-risk regions for esophageal cancer in China. So we believe that our data may provide a better understanding of ESCC in Xinjiang. Until now, few large cohort studies focused on postoperative adjuvant therapy of ESCC in Xinjiang, our results will be useful for the whole management of esophageal cancer. Of course, further follow-up studies are required to confirm our findings and develop new therapeutic strategies for these patients.

\section{Conclusion}

Adjuvant therapy of these patients with ESCC was shown to be insufficient. Enhanced treatment after surgery needs further improvement. Health sector should implement targeted treatment regime for different areas to alleviate the burden of cancer.

\section{Declarations}

\section{Ethics approval}

This study was approved by the medical ethics committee of Affiliated Tumor Hospital of Xinjiang Medical University.

\section{Consent to Participate}

A waiver for informed consent was granted by the medical ethics committee of Affiliated Tumor Hospital of Xinjiang Medical University.

\section{Consent for publication}

Not Applicable.

\section{Data Availability Statement}

The data that support the findings of this study can be available from the corresponding author upon reasonable request.

\section{Authors' contributions}

we confirmed that all authors have contriubted to and agreed on the content of the manuscript, YLF drafted the manuscript and contributed all tables and figures shown. LT helped analyze the data. ZST 
and LQ provided suggestions in the discussion. ZQQ, HXJ and TA offered some help in the preparation of manuscript. LXM conceived and took charge of the whole study.

\section{Funding}

The study was funded by the Natural Science Foundation of China (81960527, U1603284, 81860511), Key Research and Development Project of the Xinjiang Uygur Autonomous Region (2020B03003-1), Science and Technology Projects of Xinjiang Uygur Autonomous Region (2018E02067), TianshanXuesong Project of the Xinjiang Uygur Autonomous Region (2018XS19), General Project from State Key Laboratory of Pathogenesis, Prevention, Treatment of High Incidence Diseases in Central Asia, Urumqi, Xinjiang Uygur Autonomous Region (SKL-HIDCA-2020-11,12) and Natural Science Foundation of Xinjiang Uygur Autonomous Region (2020D01C218).

\section{References}

1. Bray F, Ferlay J, Soerjomataram I et al: Global cancer statistics 2018: GLOBOCAN estimates of incidence and mortality worldwide for 36 cancers in 185 countries. CA: a cancer journal for clinicians 2018, 68(6):394-424.

2. Zhao $X$, Tang $Y$, Wang $C$ et al: Prognostic values of STAT3 and HIF-1a in esophageal squamous cell carcinoma. European review for medical and pharmacological sciences 2019, 23:3351-3357.

3. Lagergren J, Smyth E, Cunningham D et al: Oesophageal cancer. The Lancet 2017, 390(10110):23832396.

4. DeMeester SR: Epidemiology and biology of esophageal cancer. Gastrointestinal cancer research: GCR 2009, 3(2 Suppl 1):S2.

5. Dong J, Thrift AP: Alcohol, smoking and risk of oesophago-gastric cancer. Best Practice \& Research Clinical Gastroenterology 2017, 31(5):509-517.

6. Jia A, Wu Y, Ren W et al: Genetic variations of CARMN affect risk of esophageal cancer in northwest China. Gene 2020, 748:144680.

7. Chen W, Zheng R, Baade PD et al: Cancer statistics in China, 2015. CA: a cancer journal for clinicians 2016, 66(2):115-132.

8. Herskovic A, Russell W, Liptay M et al: Esophageal carcinoma advances in treatment results for locally advanced disease. Annals of oncology 2012, 23(5):1095-1103.

9. Kurokawa Y, Sasako M: The Asian Perspective on the Surgical and Adjuvant Management of Esophagogastric Cancer. Surgical Oncology Clinics of North America 2017, 26(2):213-224.

10. Zhang H, Tang P, Miao $X$ et al: Does tumor size improve the accuracy of prognostic prediction in patients with esophageal squamous cell carcinoma after surgical resection? Oncotarget 2016, 7(41):- 
11. Zhang L, Ma LL, Zhang JQ et al: Comparisons of Curative and Side Effects of Chemoradiotherapy among Xinjiang Han, Uigur and Kazakh Esophageal Carcinoma Patients. Asian Pacific Journal of Cancer Prevention Apjcp 2012, 13(1):169-173.

12. Ma J-Y, Wu Z, Wang Y et al: Clinicopathologic characteristics of esophagectomy for esophageal carcinoma in elderly patients. World Journal of Gastroenterology: WJG 2006, 12(8):1296.

13. Bonavina L, Incarbone R, Saino $G$ et al: Clinical outcome and survival after esophagectomy for carcinoma in elderly patients. Diseases of the Esophagus 2003, 16(2):90-93.

14. Zheng S, Vuitton L, Sheyhidin I et al: Northwestern China: a place to learn more on oesophageal cancer. Part one: behavioural and environmental risk factors. European journal of gastroenterology \& hepatology 2010, 22(8):917-925.

15. Kou K, Baade PD, Gatton M et al: Individual-and Area-Level Socioeconomic Inequalities in Esophageal Cancer Survival in Shandong Province, China: A Multilevel Analysis. Cancer Epidemiology and Prevention Biomarkers 2019, 28(9):1427-1434.

16. Alaouna M, Hull R, Penny $C$ et al: Esophageal cancer genetics in South Africa. Clinical and experimental gastroenterology 2019, 12:157.

17. Ajani JA, D'Amico TA, Bentrem DJ et al: Esophageal and esophagogastric junction cancers, version 2.2019, NCCN clinical practice guidelines in oncology. Journal of the National Comprehensive Cancer Network 2019, 17(7):855-883.

18. Lordick F, Mariette C, Haustermans K et al: Oesophageal cancer: ESMO Clinical Practice Guidelines for diagnosis, treatment and follow-up. Annals of Oncology 2016, 27(suppl_5):v50-v57.

19. Zhang B, Xiao Q, Yang D et al: Spindle cell carcinoma of the esophagus: A multicenter analysis in comparison with typical squamous cell carcinoma. Medicine 2016, 95(37).

20. Yang H, Liu H, Chen $\mathrm{Y}$ et al: Neoadjuvant chemoradiotherapy followed by surgery versus surgery alone for locally advanced squamous cell carcinoma of the esophagus (NEOCRTEC5010): a phase III multicenter, randomized, open-label clinical trial. Journal of Clinical Oncology 2018, 36(27):2796.

21. Chen S, Huang S, Liu D et al: Clinicopathological features and prognosis of esophageal squamous cell carcinoma in young patients. Diseases of the Esophagus 2019, 32(5):doy070.

22. Liu $Y$, Chen $X$, Wang $Y$ et al: Clinical features and prognostic factors for surgical treatment of esophageal squamous cell carcinoma in elderly patients. Journal of BU ON: official journal of the Balkan Union of Oncology 2019, 24(3):1240-1244.

23. Prabhu A, Obi KO, Rubenstein JH: The synergistic effects of alcohol and tobacco consumption on the risk of esophageal squamous cell carcinoma: a meta-analysis. American Journal of Gastroenterology 2014, 109(6):822-827.

\section{Figures}




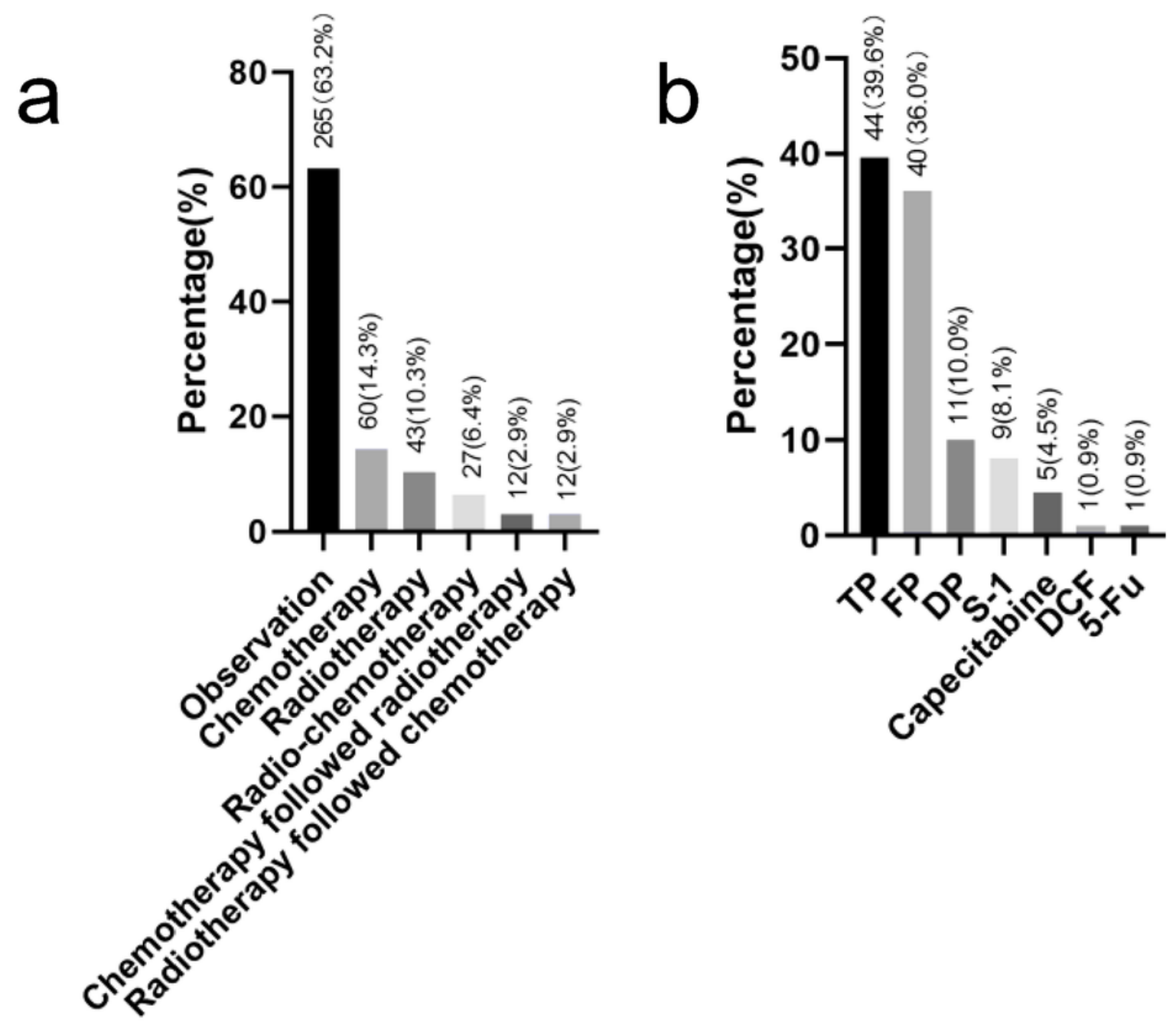

Figure 1

(a) Treatment after operation of 419 patients with ESCC. (b) Adjuvant chemotherapy regimens of 111 patients. DCF: Docetaxel , cisplatin and 5-fluorouracil ; TP: Paclitaxel and cisplatin ; DP囚Docetaxel and cispaltin; FP: 5-fluorouracil and cisplatin; P: Cispaltin; S-1: Tegafur. 


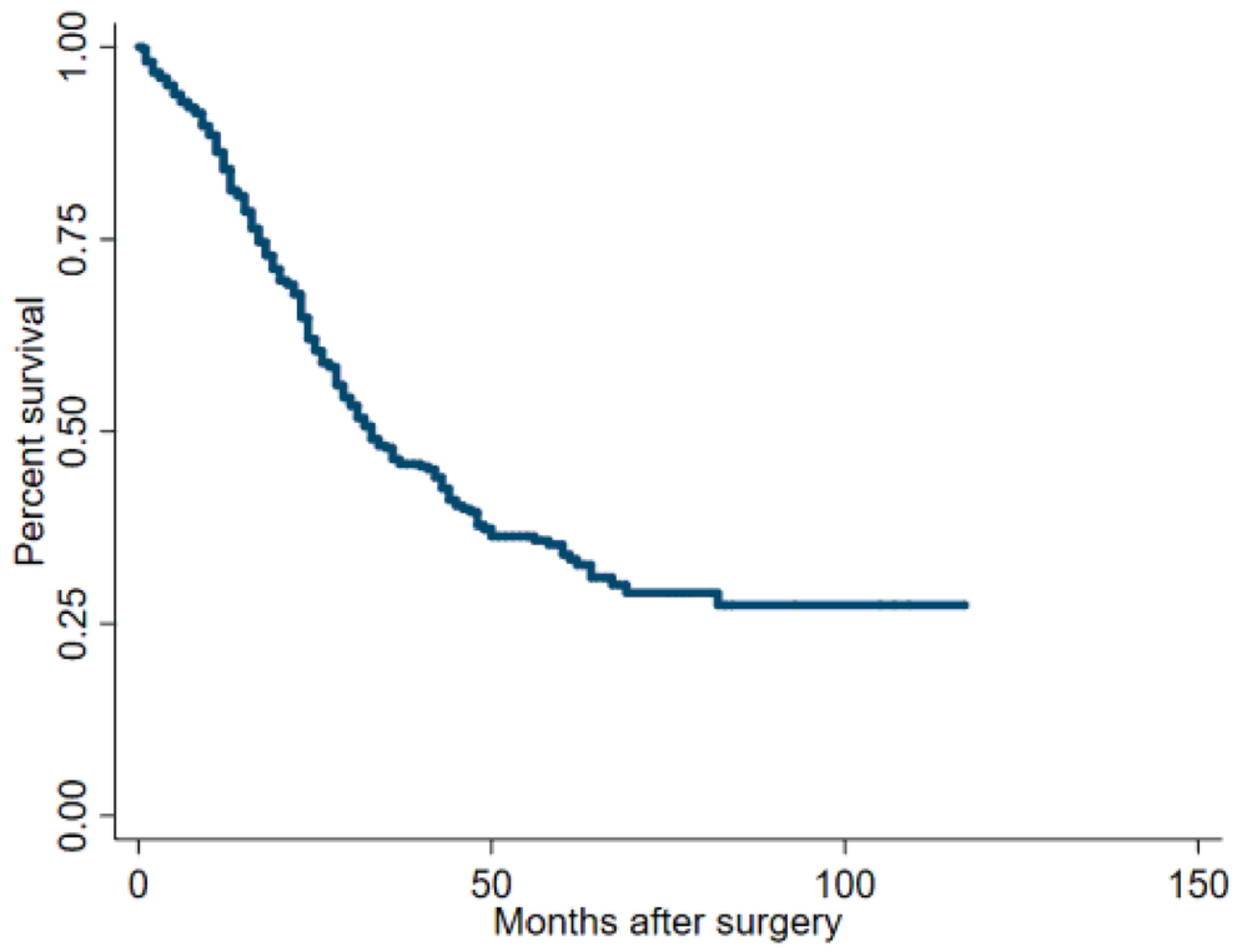

Number at risk

419

81

5

0

Figure 2

The overall survival (OS) of 419 patients of ESCC. 